\title{
Linear Receiver Design for Time-Varying Poisson Molecular Communication Channels with Memory
}

\author{
Fardad Vakilipoor ${ }^{\ddagger}$, Francesca Ratti ${ }^{\ddagger}$, Maurizio Magarini ${ }^{\ddagger}$, and Hamdan Awan* \\ ‡Dipartimento di Elettronica, Informazione e Bioingegneria (DEIB) \\ Politecnico di Milano, Milano, Italy \\ *Telecommunications Software and Systems Group (TSSG) \\ Waterford Institute of Technology, Ireland \\ Email: fardad.vakilipoor@mail.polimi.it, francesca.ratti@polimi.it, maurizio.magarini@polimi.it, hawan@tssg.org
}

\begin{abstract}
This work investigates novel approaches for designing the linear filter and the threshold of the memory-less detector in the receiver of a diffusion based molecular communication system. We focus on the case where the transmitted symbols propagate through a Poisson channel with memory that introduces inter-symbol interference. Inspired by the theory of filtering under Poisson regime and considering all the possible mean values of the interference, we propose two new design methods for computing the coefficients of the filter. While in the development of the theory under Poisson regime the interference is not considered in the design of the filter, in our proposal we take it into account in the time-variation of the mean value. Since the proposed approach gives better performance for low transmission rate, another design based on linear programming is proposed for the high-rate case. For the design of the detector's threshold we use an averaging method over the output of the receiving filter. The performance of the two proposed designs, in terms of bit error rate, is compared to that achieved by an approach which is based on the maximization of the signal-tointerference plus noise ratio (SINR). We show that our design achieves almost the same performance of the maximum SINR one with the advantage of lower computational cost to obtain receiver coefficient $s$ and threshold.
\end{abstract}

\section{INTRODUCTION}

$\mathbf{M}$ OLECULAR communication (MC) is an emerging paradigm where molecules act as the carrier of information between transmitting and receiving nanomachines. Potential applications of this multidisciplinary field are mainly in the health sector, e.g. smart drug delivery [1], health monitoring [2], etc [3]. MC can be abstracted as consisting of three main components i.e. transmitter, channel, and receiver.

A primary challenge in $\mathrm{MC}$ is to reach a reliable transmission, especially in presence of different impairments such as channel interference. A typical procedure is to categorize channels as a combination of three main characteristics, free diffusion [4], [5], advection [6], [7] and reactions [8], [9]. This paper uses a model which includes all the aforementioned characteristics. With reference to the diffusive nature of channel, the motion of information particles is stochastic. The random behavior of the molecules, in terms of their counting at the receiver, can be modeled as Bernoulli random process [10]. Moreover, existing molecules in the environment and intersymbol interference (ISI), due to the delayed arrival of previously released molecules, introduces further randomness in the received signal. Since the receiver counts the number of molecules at specific time instants, without considering where these molecules are coming from, it is required to extract the correct sequence of transmitted symbol. Therefore, the received signal is characterized by random distortions whose effect can be minimized by introducing an appropriate filtering process followed by decision on the transmitted symbols. When the number of released molecules is large and the mean of the Bernoulli random variable is small, the statistical characterization of the received signal is given as a Poisson random variable with time varying parameterization [11].

To deal with statics of the received signal, a filter design specifically intended for Poisson channel with memory is required. To this aim, in [12] two methods are proposed as "weighted sum detector", where the digital filter is defined by a moving average or has coefficients that coincide with the sampling at the symbol interval of the channel impulse response (CIR). Authors in [13] defined the signal-to-interference plus noise ratio (SINR) and proposed a filter design to maximize it. Another contribution was an analytical approach to compute the threshold used by the detector to take the decision about the most likely transmitted symbol. The proposed approach outperforms the one in [12]. However, since its design is computationally demanding, the potential application in realtime implementation is limited from a practical point of view. In this paper, we provide two different filter and threshold designs that allow us to achieve almost the same performance as that maximum SINR approach with lower number of operations to find parameters. The first design takes inspiration from the theory of filtering under Poisson regime that was developed in [14]. The second one consists in the formulation of the filtering design as a linear programming problem. Also, we consider the design of the threshold used by the memoryless detector to detect the transmitted symbol.

Rest of the paper is organized as follows. Section II describes the system model. In Sec. III we propose methods to compute linear filter coefficients. Section IV provides a new algorithm to design threshold for Poisson channel with memory. Section V compares the performance of filters together with threshold. Finally, Sec. VI presents conclusion. 


\section{SYSTEM MODEL}

This paper considers a diffusion based MC system employing on-off keying (OOK) modulation at the transmitter. In OOK, a given amount of molecules $N^{t x}$ is released to transmit symbol $s[k]=1$, which is associated to bit 1 , while no molecules are released at the corresponding time instant for $s[k]=0$, which denotes bit 0 . Symbols are assumed to be equi-probable. With reference to the diffusive channel, the effect of released molecules goes to zero asymptotically after infinite time (from a practical point of view it can be considered of finite duration). As suggested in [13] to quantify and simplify the modeling, a finite arbitrary length $L$ of the channel, measured in symbol intervals, is considered, which means the channel inherits the property of having memory. Finally, a passive receiver is assumed, which is able to count the number of molecules that are passing through without having any interaction [15]. The receiver counts the molecules from the beginning of the $k$ th symbol interval and lasts for its entire duration.

A perfect synchronization is assumed between the transmitter and the receiver, meaning that the receiver is aware of symbol timing. The received signal is uniformly sampled $M$ times in every symbol interval $T^{s y m}$. The sampling interval is $T^{c}=T^{s y m} / M$. The presence or absence of a molecule at receiver can be described by a Bernoulli random variable (RV) [5]. When the number of released molecules is large and the mean of the Bernoulli RV is small a quite accurate approximation is given by a Poisson distribution $\mathcal{P}(\lambda)$ with mean $\lambda$ [11]. However, in $T^{s y m}$ the mean varies with time. Consequently, by fixing the observation window to the $k$ th symbol interval, the number of observed molecules at the $m$ th sample, with $m=1, \ldots, M$, can be written as:

$$
r[k, m] \sim \mathcal{P}(\lambda[k, m]),
$$

where

$$
\lambda[k, m]=\sum_{l=1}^{L} \bar{c}_{s}^{(l)}[m] \cdot s[k-l+1]+\bar{c}^{e x t},
$$

is the time-varying mean and

$$
\bar{c}_{s}^{(l)}[m]=\bar{c}_{s}\left[t+(l-1) T^{s y m}\right] \cdot \operatorname{rect}\left[\frac{t-\frac{T^{s y m}}{2}}{T^{s y m}}\right] \cdot \delta\left[t-m T^{c}\right]
$$

gives the $m$ th sample of the expected number of signaling molecules within $l$ th symbol interval, with $\delta[\cdot]$ being the Kronecker delta function. Note that, to have a more realistic performance, the expected number of external interference caused by existing molecules in the medium at the receiver has been included in (2) by means of the term $\bar{c}^{e x t}$. As in [13] we set $\bar{c}^{e x t}=2$,i.e. there are two external molecules from environment inside the receiver area at each sampling time on average. The term

$$
\bar{c}_{s}(t)=\frac{N^{t x} V^{r x}}{(4 \pi D t)^{3 / 2}} \exp \left(-\mathcal{K} \bar{c}_{e} t-\frac{\left(d-v_{\|} t\right)^{2}+\left(v_{\perp} t\right)^{2}}{4 D t}\right),
$$

TABLE I: System Parameters and Values

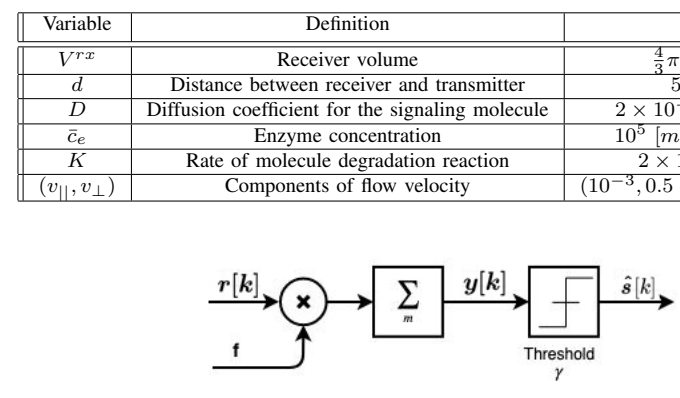

Fig. 1: Block diagram of receiver.

whose sampling appears in (3), corresponds to the expected number of observed molecules at receiver as a consequence of transmitting symbol 1 regardless of memory and interference. It is obtained by considering a three dimensional unbounded environment with uniform flow and presence of uniformly scattered enzyme molecules with degradation effect. The parameters appearing in (4) are defined in Table I, together with the numerical values that were used in the simulations. It is worth noting that (2) and (4) include the effects of other phenomena such as degradation, advection, and interference among symbols transmitted in different symbol intervals.

By reforming the expected number of observed molecules (2), the expression can be rewritten as the sum of two terms:

$$
\lambda[k, m]=\bar{c}_{s}^{(1)}[m] s[k]+\bar{c}_{i n t}[k, m],
$$

where the first term on the right-hand side accounts for the effect of expected transmitted symbol corresponding to current symbol interval while the second one gives the expected interference as $\bar{c}_{i n t}$, which depends on the previous $L-1$ transmitted symbols and $\bar{c}^{e x t}$. It is given by

$$
\bar{c}_{\text {int }}[k, m]=\sum_{l=2}^{L} \bar{c}_{s}^{(l)}[m] \cdot s[k-l+1]+\bar{c}^{e x t} .
$$

Last equation allows for designing a filter that enhances the effect of desired signal and, simultaneously, reduces the contribution coming from interference, as described in III-A.

\section{LineAR Filter DESIGN}

This section consists of three parts. First we briefly review the filter design proposed in [13]. Then, inspired by the work Bar-David in [14], we introduce a filter design algorithm that requires lower number of operations to find the coefficients. Finally, we formulate the problem of designing the linear filter into a simple linear programming approach. All the methods focus on the design of a linear filter that is applied to the $M$ samples of filter input taken in each symbol interval. By introducing the $M \times 1$ vector of real filter coefficients $\mathbf{f}=[\mathrm{f}[1], \mathrm{f}[2], \ldots, \mathrm{f}[M]]^{\top}$, being ${ }^{\top}$ the transpose operation, the output of the filter for $k$ th symbol interval is:

$$
y[k]=\mathbf{f}^{\top} \boldsymbol{r}[k],
$$

where the $M \times 1$ vector $\boldsymbol{r}[k]=[r[k, 1], r[k, 2], \ldots, r[k, M]]^{\top}$ contains the $M$ samples of the received signal in the $k$ th 


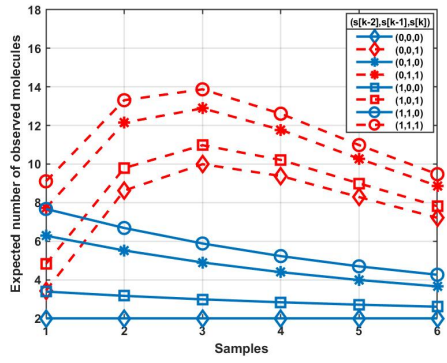

Fig. 2: Possible expected number of observed molecules in a symbol interval at sampling instants $m=1, \ldots, 6$ for memory $L=3$ and number of released molecules $N^{t x}=10^{4}$.

symbol interval. The structure of the receiver is shown in Fig. 1. In order to detect the transmitted symbol, a memoryless receiver is considered where the filter output is compared with a threshold $\gamma$ as will be explained in Sec. IV.

\section{A. Review of Maximum SINR Filter Design [13]}

The linear filter design proposed by [13] consists of the maximization of SINR that, for a generic $f$ and under the equi-probable assumption of OOK symbols, is defined as

$$
\mathrm{SINR}=\frac{0.5 \mathbf{f}^{\boldsymbol{\top}} \overline{\boldsymbol{c}}_{s} \overline{\boldsymbol{c}}_{s}^{\boldsymbol{\top} \mathbf{f}}}{\mathbf{f}^{\boldsymbol{\top}}\left(0.5 \operatorname{diag}\left\{\overline{\boldsymbol{c}}_{s}\right\}+\overline{\mathbf{C}}_{\mathbf{i n t}}\right) \mathbf{f}},
$$

where $\operatorname{diag}(\cdot)$ is a diagonal matrix whose entries are taken from elements of vector $\overline{\boldsymbol{c}}_{s}=\left[c_{s}^{(1)}[1], c_{s}^{(1)}[2], \ldots, c_{s}^{(1)}[M]\right]$ and $\overline{\mathbf{C}}_{\text {int }}$ is the covariance matrix of interference whose entries are computed as explained in the following. Let $\mathbf{s}_{i} \in\{0,1\}^{i}$ be a possible realization of a sequence of symbols over $i$ symbol intervals. For this specific sequence we denote the interference given in (6) as $\bar{c}_{\text {int }}\left[m \mid \mathbf{s}_{\mathrm{L}-1}\right]$. The entries of matrix $\overline{\mathbf{C}}_{\mathbf{i n t}}$ are computed from the following average

$$
\begin{aligned}
& \overline{\mathbf{C}}_{\mathbf{i n t}}\left(m, m^{\prime}\right)=\frac{1}{2^{L-1}} \times \\
& \begin{cases}\sum_{\forall \mathbf{s}_{\mathrm{L}-1}} \bar{c}_{i n t}\left[m \mid \mathbf{s}_{\mathrm{L}-1}\right] \bar{c}_{i n t}\left[m^{\prime} \mid \mathbf{s}_{\mathrm{L}-1}\right] \\
-\frac{1}{2^{L-1}} \sum_{\forall \mathbf{s}_{\mathrm{L}-1}} \bar{c}_{i n t}\left[m \mid \mathbf{s}_{\mathrm{L}^{-1}-1}\right] \sum_{\forall \mathbf{s}_{\mathrm{L}-1}} \bar{c}_{i n t}\left[m^{\prime} \mid \mathbf{s}_{\mathrm{L}-1}\right], & \text { if } \mathrm{m} \neq m^{\prime} \\
\sum_{\forall \mathbf{s}_{\mathrm{L}-1}} \bar{c}_{i n t}^{2}\left[m \mid \mathbf{s}_{\mathrm{L}-1}\right]+\bar{c}_{i n t}\left[m \mid \mathbf{s}_{\mathrm{L}-1}\right] & \text { otherwise. } \\
-\frac{1}{2^{L-1}}\left(\sum_{\forall \mathbf{s}_{\mathrm{L}-1}} \bar{c}_{i n t}\left[m \mid \mathbf{s}_{\mathrm{L}-1}\right]\right)^{2}, & \end{cases}
\end{aligned}
$$

The optimal filter $\mathbf{f}_{\mathrm{SINR}_{\max }}$, in the sense of SINR maximization, has the following closed form expression

$$
\mathbf{f}_{\mathrm{SINR}_{\max }}=\left(0.5 \operatorname{diag}\left\{\overline{\boldsymbol{c}}_{s}\right\}+\overline{\mathbf{C}}_{\mathbf{i n t}}\right)^{-1} \overline{\boldsymbol{c}}_{s} .
$$

\section{B. Proposed Filter Designs under Poisson Regime}

A key difficulty of the system under consideration is the memory that causes severe ISI due to delayed arrivals of molecules. The effect of ISI in a symbol interval can be clearly seen by considering the $2^{L}$ different combinations that give the mean number of observed molecules in (2). An example of the possible values assumed by the mean in the $k$ th symbol interval is shown in Fig. 2 for $L=3$ with the parameters given in Table I. Therefore, we propose to reform the problem in the context of Poisson regime communication considering that in each sampling instant the mean can assume $2^{L}$ possible values. Finally, we have a Poisson channel with time varying mean, we can employ equation (21) in [14] which designed for Poisson channel without ISI. To counter with ISI effect in channel we propose the design of two different linear filters and name them as "average" case filter, $\mathbf{f}_{\text {avg }}$ and "worst" case filter, $\mathbf{f}_{w}$.

1) "Average" Case Filter Design: The $m$ th coefficient of the receiving filter for this case is given by:

$$
\mathrm{f}_{\text {avg }}[m]=\log \left(\frac{\lambda_{(a v g, 1)}[m]}{\lambda_{(a v g, 0)}[m]}\right), m=1, \ldots, M,
$$

where $\lambda_{(a v g, 1)}[m]$ and $\lambda_{(a v g, 0)}[m]$ are sampled average of expected number of observed molecules over all permutations of sequences that represent symbols one and zero at current symbol interval that are given respectively by

$$
\begin{aligned}
\lambda_{(a v g, 0)}[m] & =\frac{1}{2^{(L-1)}} \sum_{\forall \mathbf{s}_{\mathrm{L}-1}} \bar{c}_{i n t}\left(m \mid \mathbf{s}_{\mathrm{L}-1}\right), \\
\lambda_{(a v g, 1)} & {[m]=\lambda_{(a v g, 0)}[m]+c_{s}^{(1)}(m) . }
\end{aligned}
$$

2) "Worst" Case Filter Design: By looking back at Fig. 2 it can be noticed that there are two sequences, $[0,0,1]$ as lowest one and $[1,1,0]$ as highest zero, that might be the most difficult case of detection, because they are closest realizations coming from different symbols. Generally we name these two sequences as "worst" one and "worst" zero i.e.:

$$
\begin{aligned}
& \lambda_{(w, 1)}[m]= \lambda[m \mid \underbrace{0, \ldots, 0}_{\text {previous L-1 symbols }}, \underbrace{1}_{\text {current time symbol }}] \\
& \lambda_{(w, 1)}[m]=\bar{c}_{s}^{(1)}[m]+\bar{c}^{e x t}, \\
& \lambda_{(w, 0)}[m]= \lambda[m \mid \underbrace{1, \ldots, 1}_{\text {previous L-1 symbols }}, \underbrace{0}_{\text {current time symbol }}], \\
& \lambda_{(w, 0)}[m]=\sum_{l=2}^{L} \bar{c}_{s}^{(l)}[m]+\bar{c}^{e x t} .
\end{aligned}
$$

Separating $\lambda_{(w, 1)}$ and $\lambda_{(w, 0)}$ leads to separation of the other $2^{L-1}$ realizations having one and zero in current symbol interval, thus another possible design is

$$
\mathrm{f}_{w}[m]=\log \left(\frac{\lambda_{(w, 1)}[m]}{\lambda_{(w, 0)}[m]}\right), m=1, \ldots, M .
$$

\section{Proposed Filter Design under Linear Programming}

Given the linearity of the filter, it is also possible to reframe its design into the framework of a linear programming (LP) problem subject to simple constraints. We propose a design where the objective is to maximize the distinguishability between 0 and 1 at the output of the filter. This is obtained by considering the distance between the two closest outputs according to the particular combination of transmitted symbols. Let us define the mean value vectors $\boldsymbol{\lambda}_{(w, 1)}=\left[\lambda_{(w, 1)}[1], \lambda_{(w, 1)}[2], \ldots, \lambda_{(w, 1)}[M]\right]^{\top}$ and $\boldsymbol{\lambda}_{(w, 0)}=$ $\left[\lambda_{(w, 0)}[1], \lambda_{(w, 0)}[2], \ldots, \lambda_{(w, 0)}[M]\right]^{\top}$ for the worst case of 1 and 0 , respectively. This objective is equivalent to that required by maximizing the opening of the eye diagram in digital 
transmission systems to increase immunity to the noise [16]. The objective function that maximizes the distance between two filtered mean vectors is specified as:

$$
\mathbf{f}_{L P}=\max _{\mathbf{f}}\left\{\mathbf{f}^{\boldsymbol{\top}} \boldsymbol{\lambda}_{(w, 1)}-\mathbf{f}^{\boldsymbol{\top}} \boldsymbol{\lambda}_{(w, 0)}\right\} .
$$

subject to the two following constraints:

- Constraint 1: We impose that the range of variation of the filter's output produced by all mean vectors having 1 as current symbol is bounded within a certain value $\epsilon$. The constraint is mathematically written as:

$$
\left|\mathbf{f}^{\top}\left(\boldsymbol{\lambda}_{(w, 1)}-\bar{c}^{e x t}\right)\right| \leq \epsilon
$$

The same constraint holds for all the mean vectors having 0 as current symbol, however we have not included the proof due to limited space.

- Constraint 2: In order to prevent that the filtered mean value is mapped into negative domain, we can force the filtered lowest realization to be greater than zero by imposing:

$$
\mathbf{f}^{\top} \overline{\boldsymbol{c}}^{e x t}=\bar{c}^{e x t} \sum_{m=1}^{M} \mathrm{f}[m] \geq 0,
$$

where $\overline{\boldsymbol{c}}^{e x t}=\bar{c}^{e x t} \cdot \mathbf{1}_{M, 1}$, being $\mathbf{1}_{M, 1}$ an all 1's $M \times 1$ vector.

\section{Detection}

With reference to Fig. 1, it can be seen that after the filtering operation a detection decision is required to recover the transmitted symbol. This is done by comparing the filter output with a properly designed threshold $\gamma$ by using:

$$
\hat{s}[k]= \begin{cases}1, & \text { if } y[k] \geq \gamma, \\ 0, & \text { otherwise }\end{cases}
$$

In the following subsections, we first review the design of the threshold $\gamma$ proposed by [13], which assumes that the filter output $y[k]$ is Gaussian distributed. Then, we propose a simple design of $\gamma$, which is based on the mean value of all realizations of the expected outputs.

\section{A. Gaussian approximation}

In order to derive an analytical expression for the threshold, in [13] the Gaussian approximation is introduced for $y[k] \sim \mathcal{N}\left(\mu_{i}, \sigma_{i}^{2}\right)$, where $\mu_{0}(\mathbf{s})=\mathbf{f}^{\top} \overline{\boldsymbol{c}}_{\boldsymbol{i}}(\mathbf{s}), \mu_{1}(\mathbf{s})=\mathbf{f}^{\top}\left(\overline{\boldsymbol{c}}_{\boldsymbol{s}}+\right.$ $\left.\overline{\boldsymbol{c}}_{\boldsymbol{i}}(\mathbf{s})\right), \sigma_{0}^{2}(\mathbf{s})=\mathbf{f}^{\top} \operatorname{diag}\left\{\overline{\boldsymbol{c}}_{\boldsymbol{i}}(\mathbf{s})\right\} \mathbf{f}, \sigma_{1}^{2}(\mathbf{s})=\mathbf{f}^{\boldsymbol{\top}} \operatorname{diag}\left\{\overline{\boldsymbol{c}}_{\boldsymbol{s}}+\overline{\boldsymbol{c}}_{\boldsymbol{i}}(\mathbf{s})\right\} \mathbf{f}$, and $\overline{\boldsymbol{c}}_{\boldsymbol{i}}(\mathbf{s})=\left[\bar{c}_{i}[1 \mid \mathbf{s}], \ldots, \bar{c}_{i}[M \mid \mathbf{s}]\right.$. This allows to write the probability of error as:

$$
P_{e}[k]=\frac{1}{2^{L-1}} \sum_{\forall \mathbf{s}_{\mathrm{L}-1}}\left[1-Q\left(\frac{\gamma-\mu_{1}\left(\mathbf{s}_{\mathrm{L}-1}\right)}{\sigma_{1}\left(\mathbf{s}_{\mathrm{L}-1}\right)}\right)+Q\left(\frac{\gamma-\mu_{0}\left(\mathbf{s}_{\mathrm{L}-1}\right)}{\sigma_{0}\left(\mathbf{s}_{\mathrm{L}-1}\right)}\right)\right] .
$$

The value of $\gamma$ is then numerically derived to minimize (23). This approach to find $\gamma$ has a main disadvantage of requiring the computation of $\mu_{i}(s)$ over all the realizations of $s$ that together with the computation of (23) and the numeric search of $\gamma$, makes it quite complex to compute. In next sub-section we propose a less computational demanding approach for the design of $\gamma$.

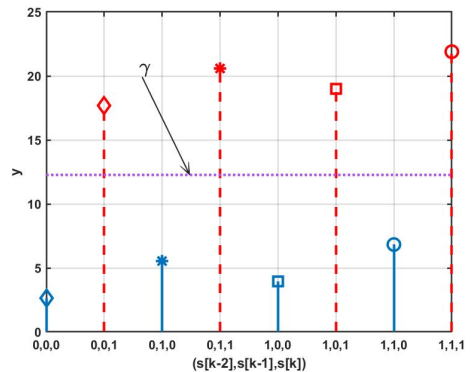

Fig. 3: All possible Expected $y$

\section{B. Mean Detection}

Figure 3 shows the possible outputs after filtering of the mean values given by (7). It can be observed that are two groups of expected realizations, i.e. dashed and solid, that can be separated by a horizontal line corresponding to the mean value of $y[k]$ conditioned on current symbol 0 and 1 , respectively. A reliable threshold that separates filter output representing ones and zeros is given as:

$$
\gamma=\frac{1}{2} \mathbf{f}^{\boldsymbol{\top}}\left(\boldsymbol{\lambda}_{(w, 0)}+\boldsymbol{\lambda}_{(w, 1)}\right) .
$$

Its derivation has not been reported here due to lack of space. Therefore in order to compute the threshold, we only need to compute "worst" one and "worst" zero vectors.

\section{Simulation Results}

The model was simulated according to the parameters in Table I. Symbol interval is computed as a multiple of peak time of $\bar{c}_{s}$ meaning, $T^{s y m}=\zeta \arg \max _{t}\left\{\bar{c}_{s}(t)\right\} . \zeta$ is a free parameter that denotes the rate of transmission, i.e. the higher is $\zeta$, the lower is the transmission rate, and we named it "Symbol Interval Factor" (SIF).

We assumed $L=3$ and $M=6$. Simulation results were obtained by averaging over transmission of $2 \cdot 10^{6}$ symbols. The performance was compared with that of [13], which is illustrated in Sec. III-A. Note that, in all the numerical results we considered both thresholds.

Figures 4 and 5 show the BER as a function of the number of released molecules for $\zeta=\{1.5,3\}$. From both the two figures we observe that by increasing the number of released molecules the BER decreases. From Fig. 4 it can be perceived that the performance of the LP filter and that of the Maximum SINR filter are close to each other, despite the computational cost of the latter is higher. Moreover, "Worst" and "Average" case filters show a poor performance. Additionally, it can be observed that the low computational cost threshold design proposed as mean detection closely matches with the algorithm proposed by [13] for the maximum SINR filter, whereas with regards to other filters it has similar performance. From Fig. 5 we observe that when $\zeta=3$, i.e. lower rate of symbol transmission and apparently lower interference pertaining to previous transmitted symbols, "Average" and "Worst" filter design methods have lower BER compared to the other two 


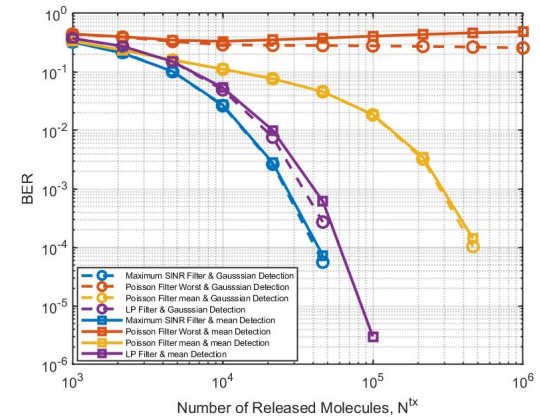

Fig. 4: BER vs. $N^{t x}, \xi=1.5$

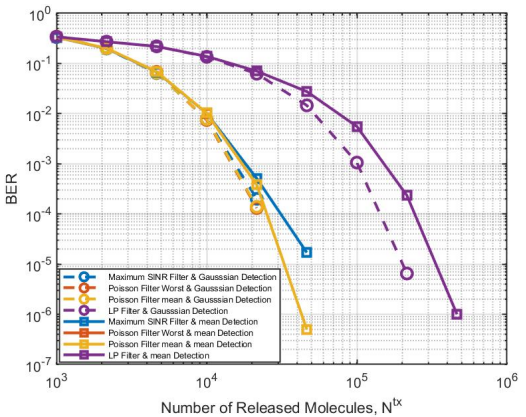

Fig. 5: BER vs. $N^{t x}, \xi=3$

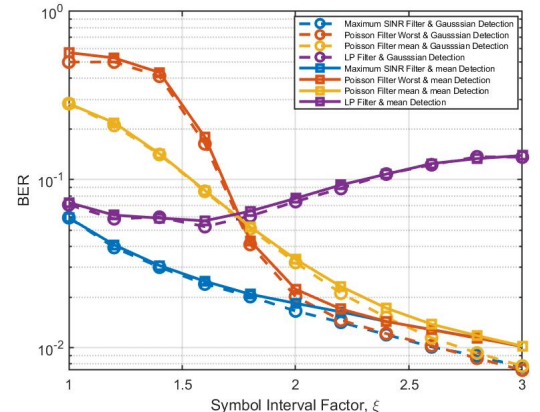

Fig. 6: BER vs. $\xi, N^{t x}=10^{4}$ approaches. Again, the BER curves of mean detection threshold behaves almost the same as the other threshold method. Finally, in Fig. 6 we set the number of released molecules to $N^{t x}=10^{4}$, and studied the BER according to the variation of SIF. For low $\zeta$, the LP filter has better performance as compared to the other two variants of the other design but it is not as good as maximum SINR filter. When $\zeta$ increases the BER of "Average" and "Worst" case filters drop to the maximum SINR one. Specifically, for $\zeta=3$ a small difference is visible between the BER curves of mean detection and Gaussian approximation detection for all but LP filter. The difference in detection arises because we did not consider the information about covariance of the random process in the design of the threshold to obtain lower computational cost.

\section{CONCLUSION}

This paper focuses on the design of the filter and detector parts in a receiver for a molecular communication (MC) system. Specifically, we delved into fresh methods to design filters and detection algorithm that take into account the computational cost with the aim of making possible the use in a scenario with real-time processing. The MC channel we considered has an unbounded environment with 3-D geometry including the effect of advection and molecule degradation, which results into a Poisson channel with memory.

Regarding the filter design, we took inspiration from the theory of Poisson filter. This shows a remarkable performance, despite its simplicity to compute the filter coefficients and threshold, in the scenario where the transmission rate is low. On the other hand, for high transmission rate its performance worsens. Therefore, we designed a method to transform our study case into a linear programming problem, which showed quite reasonable BER compared to other methods. With regards to the detection part we proposed a threshold equal to the average of filter output assuming the equal probability over all possible permutations of transmitted symbol sequences. We showed that the mean detection algorithm has almost the same performance as the one proposed by [13] with significantly lower computational cost. It is worth mentioning that the simple operations to compute the filter coefficients and threshold brings the opportunity to use these novel approaches in case of online computation for time varying channels.

\section{REFERENCES}

[1] Mauro Femminella, Gianluca Reali, and Athanasios V Vasilakos. A molecular communications model for drug delivery. IEEE transactions on nanobioscience, 14(8):935-945, 2015.

[2] Youssef Chahibi and Ilangko Balasingham. An intra-body molecular communication networks framework for continuous health monitoring and diagnosis. In 2015 37th Annual International Conference of the IEEE Engineering in Medicine and Biology Society (EMBC), pages 4077-4080. IEEE, 2015.

[3] Tadashi Nakano, Michael J Moore, Fang Wei, Athanasios V Vasilakos, and Jianwei Shuai. Molecular communication and networking: Opportunities and challenges. IEEE transactions on nanobioscience, 11(2):135148, 2012.

[4] Ling-San Meng, Ping-Cheng Yeh, Kwang-Cheng Chen, and Ian F Akyildiz. On receiver design for diffusion-based molecular communication. IEEE Transactions on Signal Processing, 62(22):6032-6044, 2014.

[5] Vahid Jamali, Arman Ahmadzadeh, Christophe Jardin, Heinrich Sticht, and Robert Schober. Channel estimation for diffusive molecular communications. IEEE Transactions on Communications, 64(10):4238-4252, 2016.

[6] Adam Noel, Karen C Cheung, and Robert Schober. Diffusive molecular communication with disruptive flows. In 2014 IEEE International Conference on Communications (ICC), pages 3600-3606. IEEE, 2014.

[7] Wayan Wicke, Arman Ahmadzadeh, Vahid Jamali, Harald Unterweger, Christoph Alexiou, and Robert Schober. Magnetic nanoparticle-based molecular communication in microfluidic environments. IEEE transactions on nanobioscience, 18(2):156-169, 2019.

[8] David Schnoerr, Ramon Grima, and Guido Sanguinetti. Cox process representation and inference for stochastic reaction-diffusion processes. Nature communications, 7(1):1-11, 2016.

[9] Akif Cem Heren, H Birkan Yilmaz, Chan-Byoung Chae, and Tuna Tugcu. Effect of degradation in molecular communication: Impairment or enhancement? IEEE Transactions on Molecular, Biological and Multi-Scale Communications, 1(2):217-229, 2015.

[10] Tadashi Nakano, Andrew W Eckford, and Tokuko Haraguchi. Molecular communication. Cambridge University Press, 2013.

[11] Athanasios Papoulis and Random Variables Probability. Stochastic Processes, volume 1065. McGraw-Hill, 1955.

[12] Adam Noel, Karen C Cheung, and Robert Schober. Optimal receiver design for diffusive molecular communication with flow and additive noise. IEEE transactions on nanobioscience, 13(3):350-362, 2014.

[13] Vahid Jamali, Arman Ahmadzadeh, and Robert Schober. On the design of matched filters for molecule counting receivers. IEEE Communications Letters, 21(8):1711-1714, 2017.

[14] Israel Bar-David. Communication under the poisson regime. IEEE Transactions on Information Theory, 15(1):31-37, 1969.

[15] Vahid Jamali, Arman Ahmadzadeh, Wayan Wicke, Adam Noel, and Robert Schober. Channel modeling for diffusive molecular communication - a tutorial review. Proceedings of the IEEE, 107(7):1256-1301, 2019.

[16] John R Barry, Edward A Lee, and David G Messerschmitt. Digital communication. Springer Science \& Business Media, 2012. 